\title{
Análisis de transparencia prenegociación en los calces OTC de los contratos de futuros sobre TRM, del mercado de derivados colombiano
}

\author{
Javier Hernando Sandoval Archila*
}

* Doctor en Ingeniería de Sistemas. Docente-Investigador, CIPE-ODEON, Universidad Externado de Colombia. Bogotá (Colombia).javier.sandoval@uexternado.edu.co

Fecha de recepción: 15 de octubre de 2016.

Fecha de aceptación: 10 de diciembre de 2016.

Para citar este artículo

Sandoval, J. H. (2016). Análisis de transparencia prenegociación en los calces отC de los contratos de futuros sobre TRM, del mercado de derivados colombiano. ODEON, 11, pp. 101-121. DOI: https://doi.org/10.18601/17941113.n11.05 



\section{Introducción}

El mercado estandarizado de derivados con subyacente USD/COP (Futuro de TRM), nace en Colombia de la mano de la Bolsa de Valores de Colombia (BVC), hace aproximadamente 10 años. Los futuros sobre TRM se dividen en futuros TRM y futuros TRS, siendo estos últimos el equivalente a los contratos mini internacionales. Las características establecidas por la BVC para los futuros de TRM y TRS se presentan en la tabla 1.

Después de diez años de existencia del mercado de derivados estandarizado, el objetivo de este trabajo es analizar el grado de transparencia del mercado de futuros sobre la TRM. Específicamente, este artículo se enfocará en los efectos de la asimetría de información que caracteriza un mercado financiero cuando las operaciones son realizadas entre agentes informados y usuarios sin acceso a la información en tiempo real. El análisis solo abarcará los futuros de TRM que cuentan con un subyacente de US 50.000 .

Según Harris (2002), la transparencia de mercado se puede dividir en dos clases: transparencia prenegociación y transparencia posnegociación. La primera es aquella que se relaciona con la información necesaria para tomar la decisión de compra o venta, por ejemplo, el estado del libro de órdenes y mejores puntas de mercado. Por otro lado, la segunda hace referencia al reporte de volúmenes y precios una vez la decisión de calce se ha tomado. Así, un mercado transparente será el que ofrezca transparencia prenegociación y posnegociación.

Hay que destacar que a pesar de ser productos similares, los futuros sobre TRM y los forwards sobre USD/COP se diferencian ampliamente en términos de transparencia. El mercado forward no cuenta con información prenegociación centralizada y, además, las negociaciones se realizan de manera bilateral sin necesidad de pasar por una bolsa organizada. Por tanto, este estudio solo se centrará en los contratos de futuros que son los únicos que cuentan con información prenegociación accesible para cualquier participante de mercado en forma de ofertas de compra y venta consignadas en el libro de órdenes. Los contratos forward son opacos por definición en términos de transparencia prenegociación.

La experiencia internacional en el mercado europeo y norteamericano presenta un mercado de futuros totalmente transparente para los usuarios del sistema electrónico de negociación. Los usuarios tienen acceso en tiempo real a las órdenes consignadas en el libro de órdenes y las operaciones son informadas de manera inmediata por el sistema transaccional (ISDA, 2009). Por tanto, la transparencia se asegura evitando las transacciones por fuera del sistema de calce 
automático y asegurando el acceso igualitario a la información de mercado, al menos para los usuarios del sistema. En general, la transparencia en los mercados financieros es vista por los reguladores como un elemento favorable para el desarrollo financiero y la protección del cliente final, por ejemplo (EC, 2006).

En el caso de los futuros de TRM del mercado colombiano existe una regulación clara respecto a transparencia posnegociación. Por un lado, está el artículo 2.1.6 de la circular única de derivados de la B VC que habla sobre la transparencia en la formación de precios. Los operadores de mercado, bajo el principio de lealtad en la celebración y registro de operaciones en el sistema, deberán abstenerse de realizar operaciones de compra o venta, que tengan por objeto, entre otros, desorganizar el mercado de derivados, falsear la libre formación de precios y manipular las cotizaciones.

Debido a que los futuros estandarizados son incluidos dentro de la definición de valor que da la Ley 964 de 2005, la Circular Externa 19 de 2008 en su artículo 3.2.1 establece la obligatoriedad de reportar las transacciones realizadas antes de los 15 minutos siguientes a la ejecución del calce.

El mercado de derivados estandarizado está en línea con los tiempos de requerimiento de registro en el activo subyacente. Para el caso de operaciones en el mercado Spot del dólar, el tiempo máximo para registrar una operación será de 15 minutos contados desde la creación del calce. Este tiempo lo estipula la regulación cambiaria del Banco de la Repúbica, consignada en la Circular Reglamentaria Externa $D O D M-317$ que reglamenta la resolución externa 4 de 2009 y el punto 2.3.1 del Boletín 14 del 30 de abril de 2013.

Por otro lado, la normatividad no menciona la obligación de los intermediarios de mercado de revelar la información disponible a quien no tiene acceso directo. Por tanto, se asume que la transparencia prenegociación, propia de un mercado de calce automático, solo cobija a los agentes con acceso al mercado, es decir, a los intermediarios financieros y a los clientes profesionales que pagan por el servicio de precios de Bloomberg o Thomson-Reuters. Así, podemos llamar al mercado de futuros sobre TRM colombiano un mercado transparente en términos posnegociaciación y de transparencia condicional en términos de transparencia prenegociación.

Bajo este contexto, el objetivo de este trabajo se centra en realizar un analisis empírico de la transparencia prenegociación para el mercado de futuros TRM del mercado colombiano, haciendo especial énfasis en las operaciones realizadas entre intermediarios profesionales y terceros (clientes). Este análisis se da teniendo 
en cuenta que más del $99 \%^{1}$ de los calces reportados en el sistema son producto de registro de operaciones realizadas por fuera del sistema transaccional.

\begin{tabular}{|l|c|c|}
\hline & TRS & TRM \\
\hline Tamaño contrato & USD 5.000 & USD 50.000 \\
\hline Vencimientos & \multicolumn{2}{|c|}{ H,M,U,Z (4) y 2 contratos mensuales } \\
\hline Tick de Precio & \multicolumn{2}{|c|}{0,1} \\
\hline Método de Liquidación & \multicolumn{2}{|c|}{ Financiero } \\
\hline Ultimo día Negociación & \multicolumn{2}{c|}{ Segundo miércoles del mes de vencimiento } \\
\hline Garantía sobre posición & $8 \%$ \\
\hline
\end{tabular}

Tabla 1: Generalidades de los contratos de futuros estandarizados de la Bolsa de Colombia. Fuente: BVC.

\section{Datos}

La serie de datos estudiada corresponde a los libros de órdenes de compra y venta de los contratos de futuros TRMX13F y TRMZ13F con intervalos de captura de 10 segundos y los calces realizados durante los 14 días transables comprendidos entre el 29/10/2013 y el 19/11/2013. Los datos fueron tomados de las entregas que realiza la BVC por su sistema electrónico de información, $S A E$. De la serie original de calces, fueron inicialmente eliminados aquellos que se realizaron después de la 1:00:00 p.m. dado que la liquidez del libro de futuros sobre TRM cae significativamente después de ese momento.

Durante el periodo estudiado se calzaron 7.009 contratos en el TRMZ13F y 8.591 contratos en el TRMX13F. De los anteriores contratos, solo se tomaron 5.430 y 8.180 contratos respectivamente del TRMZ13F y del TRMX13F que cumplían la regla de tiempo expuesta en el párrafo anterior. Así, durante la franja de tiempo estudiada se negociaron 680 millones de dólares. Es importante notar que de esta cifra, 118 contratos usaron el motor de calce de la bolsa. El $99 \%$ de los contratos negociados se tranzaron OTC.

Para el caso de la información de mercado disponible durante los calces en los 14 días estudiados, se tiene una serie de datos de 20.275 y 25.980 actualizaciones del libro de órdenes tanto de compra como de venta para los contratos $T R M X 13 F$ y $T R M Z 13 F$ respectivamente.

Este estudio comparará los calces que se realizaron con la información prenegociación disponible en el mercado. Los calces que se dieron con ausencia de

${ }^{1}$ Cálculos propios basados en la información de mercado provista por la BVC. 
órdenes en el libro de mercado, o con presencia de libro de órdenes con una profundidad menor a la de la cantidad calzada, fueron adicionalmente separados del estudio.

\section{Modelo}

El estudio planteará la siguiente hipótesis de trabajo para abordar el problema de transparencia prenegociación en los contratos de futuros sobre TRM.

\subsection{Hipótesis}

"Los operadores escogen realizar transacciones por fuera del sistema electrónico porque pueden fijar una utilidad mayor en la transacción contra agentes que no tienen acceso a la información prenegociación, en este caso, el libro de órdenes de los contratos de futuros".

Para la verificación de esta hipótesis de trabajo, se comenzará construyendo la información de mercado disponible para el momento del calce registrado. Primero se construirá el libro de órdenes para el momento del registro de los calces. Dado que las operaciones OTC sobre futuros de TRM cuentan con $15 \mathrm{mi}-$ nutos de espacio para ser registradas, se tendrán en cuenta las puntas de compra y venta de mercado disponibles para todo el intervalo de tiempo permitido por la regulación vigente. Adicionalmente, las órdenes de mercado se ponderarán por volumen para que se cuente con los precios de mercado equivalentes al volumen negociado.

La representación del tiempo de espera se modelará siguiendo una distribución Gamma con parámetros de localización 7 y de escala 1.3. De esta forma, el modelo asegura que con el $95 \%$ de confianza, el tiempo transcurrido desde la transacción al registro sea de mínimo 4 y máximo 15 minutos (figura 1). Tiempos por encima de 20 minutos se vuelven, desde el punto de vista de la probabilidad de ocurrencia, despreciables. Adicionalmente, y como forma de capturar el caso más extremo posible, se asumirá que el calce de la operación se realizó cuando estaban vigentes los precios más extremos posibles en la ventana de tiempo anterior de 15 minutos. Por tanto, se asumirá que las ofertas de compra se encontraban en su punto más bajo del intervalo y las ofertas de venta en el punto más alto. Esta metodología de captura del tiempo de espera está en línea con la obligación de los operadores de registrar sus operaciones en un tiempo que no supere los 15 minutos.

pp. 101-121 • n. ${ }^{\circ} 11 / 2016$ 
De esta forma, el precio de referencia de mercado basado en la información prenegociación será calculado usando la información de precios disponibles (antes del registro de la operación) ponderados mediante las dos técnicas mencionadas anteriormente.

Para el método que denominaremos $\operatorname{Gamma}(7,1.3)$, los precios de referencia serán:

$$
\begin{aligned}
& p_{i}^{\bar{c} G}=\sum_{i=1}^{20 * 6} p^{c}(t-i) \Delta G(i-1, i), \\
& p_{i}^{\bar{v} G}=\sum_{i=1}^{20 * 6} p^{v}(t-i) \Delta G(i-1, i),
\end{aligned}
$$

con $p^{c}(t-i)$ como el precio de compra ${ }^{2}$ ponderado por volumen del calce $i$ disponible durante los instantes $(t-i-1, t-1)$ anteriores al registro de la operación; $i$ podrá tomar valores hasta 120 porque la unidad de tiempo es 10 segundos y el intervalo total considerado es 20 minutos. $\Delta G(i-1, i)$ corresponderá a la probabilidad de observar un registro en el intervalo $(t-i-1, t-i)$ siguiendo la distribución de probabilidad Gamma que describe el tiempo de espera desde el calce al registro. En otras palabras, la probabilidad de que el intermediario se demore entre $i-1$ e $i$ segundos en realizar el registro, contado a partir del momento del calce. $p_{i}^{\bar{c} G}$ y $p_{i}^{\bar{v} G}$ se denominarán precio de mercado ponderado por tiempo de arribo del registro en compra y venta respectivamente utilizando la metodología $\operatorname{Gamma}(7,1.3)$.

\footnotetext{
${ }^{2} \mathrm{El}$ precio de compra disponible en el mercado ha sido ponderado por volumen para tener cantidades equivalentes al calce observado. Esto también aplica para los precios de venta.
} 


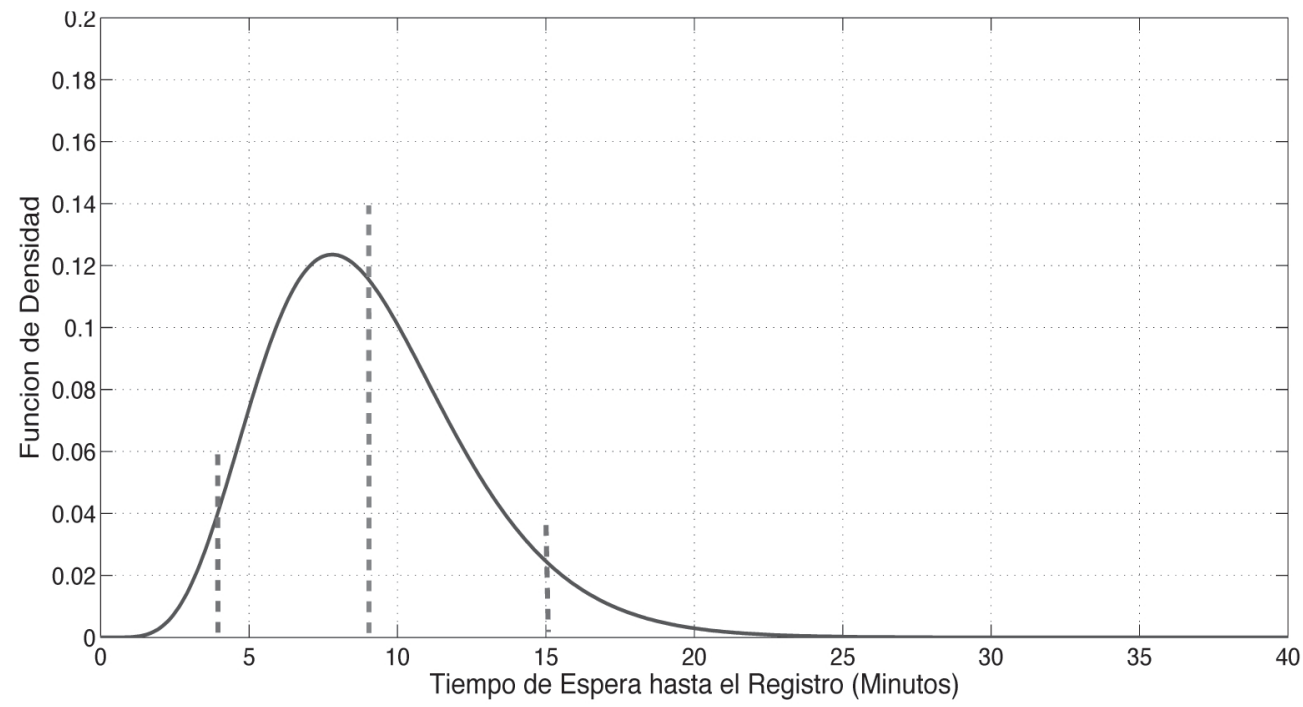

Figura 1: Modelación del tiempo de espera para el registro desde el tiempo de la transacción. La distribución Gamma ha sido calibrada para que el $95 \%$ de las observaciones se ubiquen entre 4 y 15 minutos. La línea punteada central representa la media y las dos líneas punteadas laterales representan el intervalo de $95 \%$ de confianza.

Para el método MinMax que considera que la transacción se realizó en el momento más extremo del libro de órdenes, se tendrá que:

$$
\begin{aligned}
& p_{i}^{\bar{c} M}=\operatorname{Min} p^{c}(t-i), i=1 \ldots, 6 * 15, \\
& p_{i}^{\bar{v} M}=\operatorname{Max} p^{v}(t-i), i=1 \ldots, 6 * 15,
\end{aligned}
$$

con $p_{i}^{\bar{c} M}$ como el precio de compra más bajo en el intervalo de tiempo correspondiente a 15 minutos anteriores al registro y $p_{i}^{\bar{v} M}$ como el precio de venta más alto en el intervalo de tiempo correspondiente a 15 minutos anteriores al registro. Por tanto, los precios de referencia se escogen para capturar las condiciones extremas de negociación durante el intervalo de tiempo transcurrido entre el calce y el registro.

Dado que la mayoría de las operaciones de mercado son registros, si la hipótesis estudiada es falsa, se esperaría encontrar los precios de calce dentro de los límites establecidos por los modelos anteriores. Calces que se encuentren por fuera, serán considerados transacciones realizadas a precios peores que los 
ofrecidos por el mercado. Así:

$$
p_{i}^{\bar{c} G} \leq p_{i}^{\text {calce }} \leq p_{i}^{\bar{v} G}
$$

para el caso del método Gamma(7,1.3) y

$$
p_{i}^{\bar{c} M} \leq p_{i}^{\text {calce }} \leq p_{i}^{\bar{v} M}
$$

para el caso del método MinMax; $i$ representa cada uno de los calces estudiados.

Intuitivamente, si el precio de un registro de operación $p_{i}^{\text {calce }}$ se encuentra dentro del intervalo generado por el modelo $\operatorname{Gamma}(7,1.3)$ o el modelo MinMax, el precio negociado por fuera del motor de calce de la BVC está en línea con los mejores precios otorgados por el mercado transaccional. Si un registro de operación $p_{i}^{\text {calce }}$ se encuentra por fuera del intervalo, se evidenciará un precio de negociación en peores condiciones que las ofrecidas por los agentes del mercado a través del sistema transaccional.

La figura 2 muestra un ejemplo de la localización de los límites calculados en las ecuaciones 3 y 4 para un registro particular. En el ejemplo observado, el precio de registro, identificado con una estrella en la parte izquierda de la figura, no viola el rango para ninguno de los métodos.

Las figuras 3 y 4 muestran los límites calculados usando el método Gamma(7,1.3) junto a los calces registrados durante el intervalo de estudio para los contratos TRMX13F y TRMZ13F. 


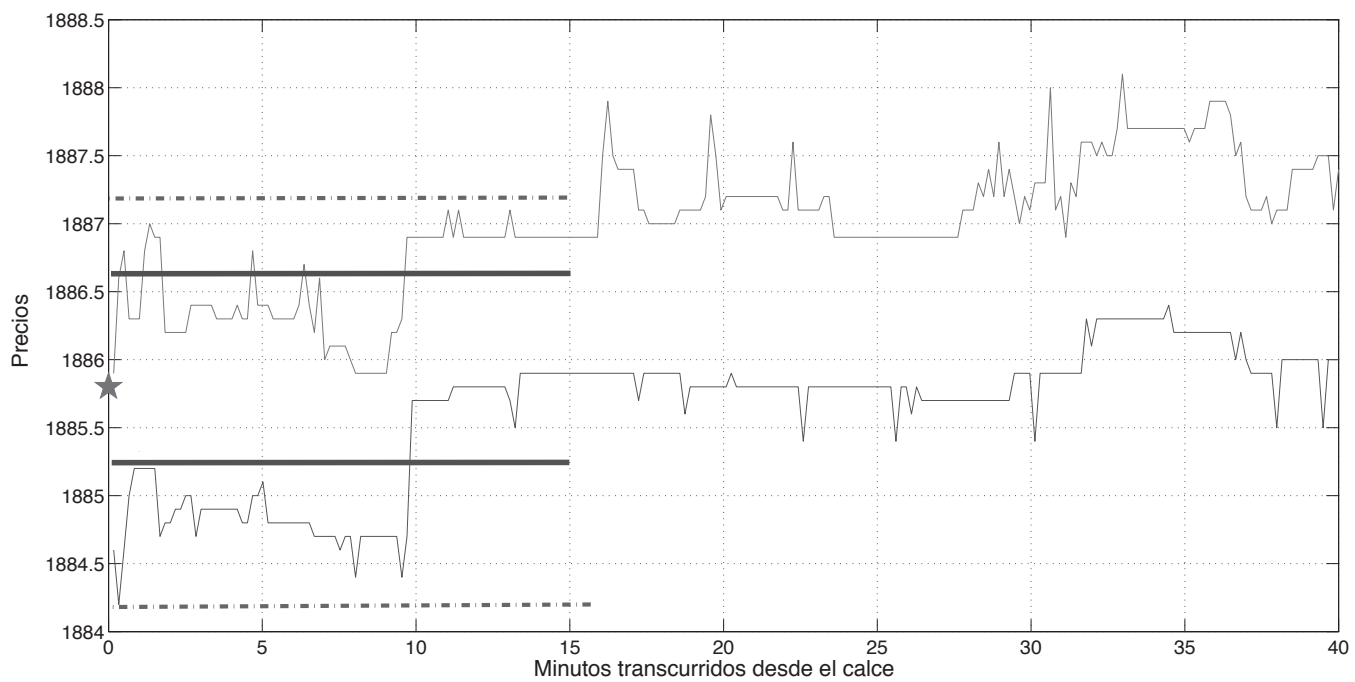

Figura 2: Ejemplo de construcción de las mejores puntas comparativas de compra y venta por el método MinMax y el método Gamma(7,1.3). La estrella representa el registro. Las líneas externas representan las mejores puntas de compra y venta usando MinMax. Las líneas internas representan las mejores puntas de compra y venta por el método Gamma(7,1.3). El eje $X$ representa minutos transcurridos desde el calce hasta el registro.

\section{Resultados}

Para el estudio de la hipótesis planteada se evaluaron los límites expuestos en las ecuaciones 3 y 4 durante la franja de datos estudiada. Debido a que las operaciones por fuera del mercado transaccional tienen costos diferentes a las transacciones realizadas por el motor de calce, el primer paso fue calcular el costo de transacción de operaciones OTC en futuros de TRM. La tabla 2 resume los costos variables en los que un intermediario del mercado de futuros tiene que incurrir para otorgar liquidez a los contratos TRM sin exponerse a riesgo de mercado. El apéndice A identifica la metodología del cáculo de costos.

Por cada 250 mil dólares, el intermediario deberá cubrir su riesgo de tipo de cambio y su riesgo de tasa de interés. La primera cobertura implicará una transacción en el mercado del dólar Spot a un costo aproximado de 10.191 pesos. La segunda cobertura le significa operar un swap Dolar Spot-Futuro, lo cual tiene un costo de 10.191 pesos en el spot y 21.645 pesos en el futuro. Por último, registrar la transacción original sobre el TRM le supondrá un costo de 21.645 


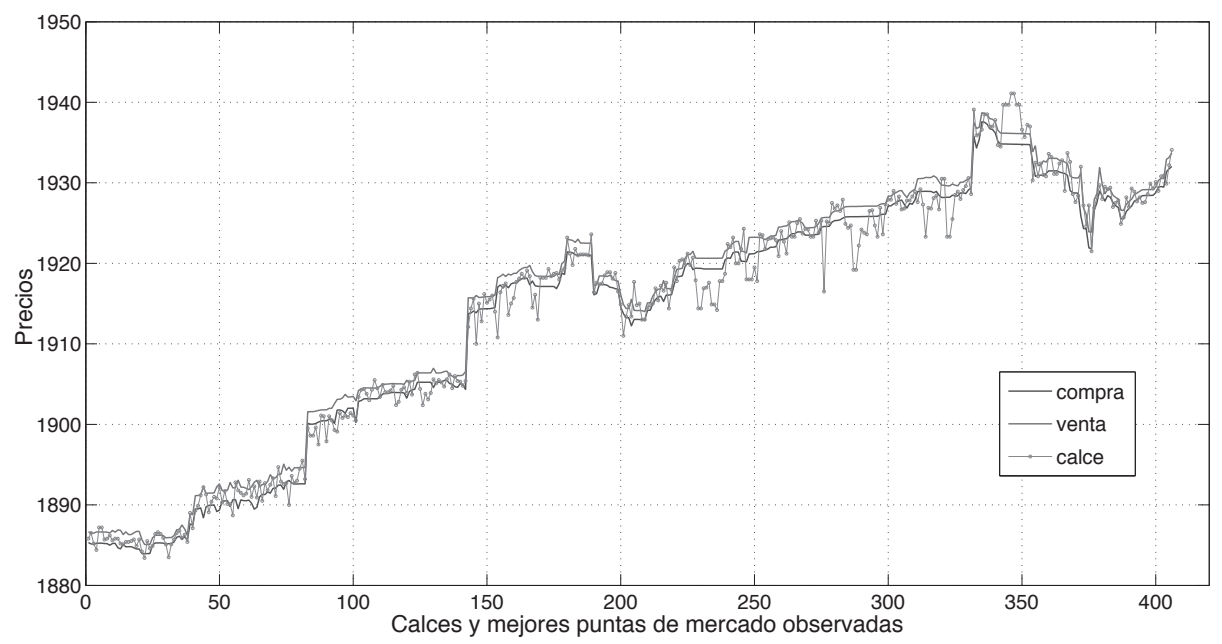

Figura 3: Evolución de las mejores puntas y los calces en el contrato TRMX13F desde el 29/10/2013 hasta el 19/11/2013. Las mejores puntas han sido ponderadas por volumen para coincidir con el volumen del calce. El método de cálculo de las mejores puntas es $\operatorname{Gamma}(7,1.3)$.

pesos. El total de costos variables para ofrecer liquidez sin riesgo en el futuro de TRM por fuera del mercado será de 63.672 pesos por cada 250 mil dólares o 0,254 pesos por dólar. Esta cifra anterior solo cubre costos variables del negocio. Asumiendo que el operador tiene un flujo mensual de negociación de 50 millones de dólares, y que sus costos fijos (costos plataformas transaccionales, entre otros) suman 5 millones de pesos mensuales, los costos variables deberán ser ajustados en 0,1 centavos por dólar. Así, el costo total de otorgar liquidez por fuera de mercado a los futuros de TRM ha sido calculado en 0,36 pesos por dólar operado.

Dado que ya se cuenta con el cáculo de costos cuando un intermediario decide otorgar liquidez fuera del sistema a los futuros de TRM, se decidió incorporar el costo de transacción en los límites evaluados. De esta forma, solo serán contadas las violaciones de los límites que sobrepasen los costos de transacción calibrados en 0,36 centavos de dólar por cada contrato registrado. Este espacio adicional eliminará los costos de transacción para justificar precios por fuera de mercado.

A partir de ahora, el término sobrecosto hará referencia a la distancia del precio del calce por encima (debajo) de los precios de referencia calculados según la ecuación (2), teniendo en cuenta 0,36 pesos por dólar por concepto de costos de transacción. 


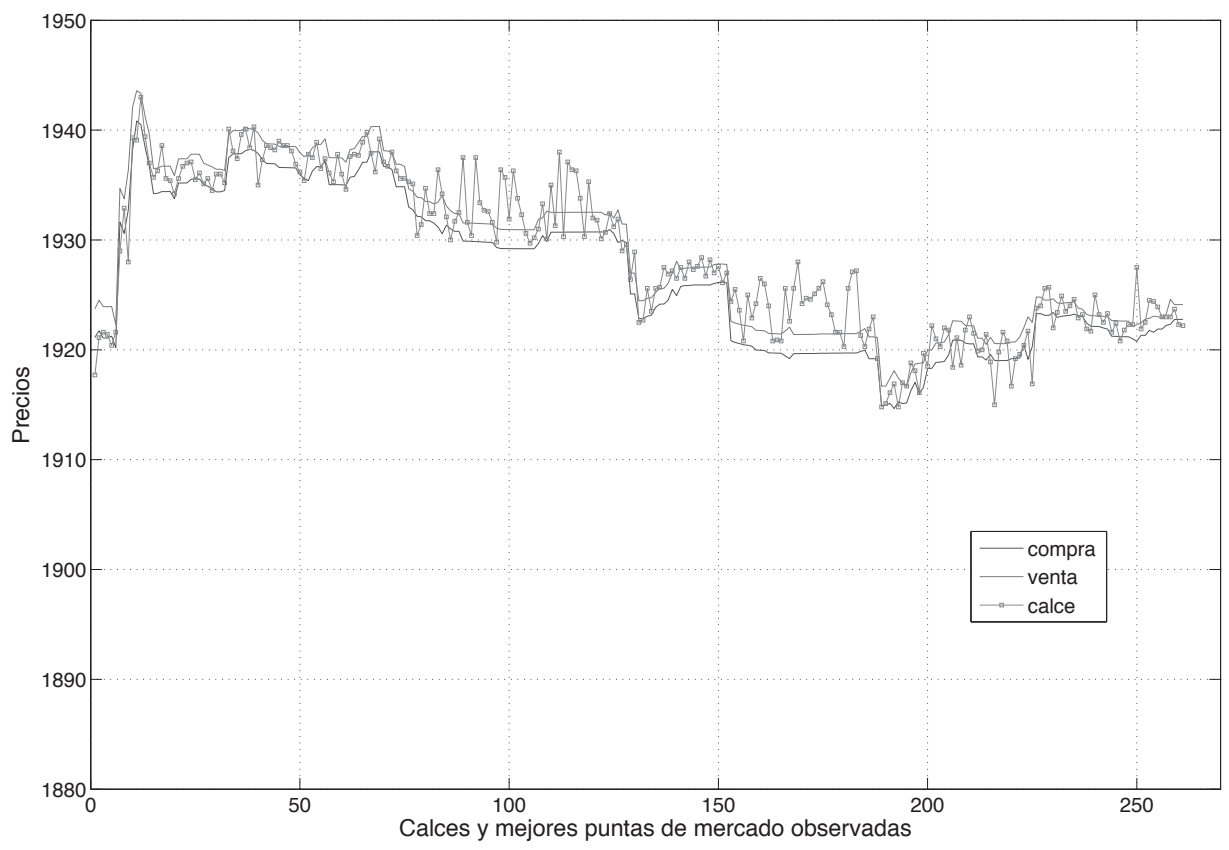

Figura 4: Evolución de las mejores puntas y los calces en el contrato TRMZ13F desde el 29/10/2013 hasta el 19/11/2013. Las mejores puntas han sido ponderadas por volumen para coincidir con el volumen del calce. El método de cálculo de las mejores puntas es $\operatorname{Gamma}(7,1.3)$.

Primero se expondrán los resultados al utilizar el método $\operatorname{Gamma}(7,1.3)$. Durante los 14 días estudiados, solo 9.577 contratos negociados cumplieron la condición de poseer libro de órdenes para todo el intervalo anterior de 20 minutos. Por tanto, el resto de los calces fueron rechazados. De los calces estudiados, $30 \%$ estuvieron por fuera de mercado con un valor de sobrecosto promedio de 1,91 pesos por dólar y valores mínimos y máximos de 0,36 y 8,23 pesos por dólar respectivamente.

La figura 5 muestra la frecuencia de los sobrecostos y la relación de sobrecostos en función del tamaño del contrato negociado. Como se puede evidenciar, el sobrecosto más común es de alrededor de 1 peso y se da con mayor frecuencia en calces de 10 o menos contratos. Sin embargo, los sobrecostos llegaron a niveles superiores a los 7 pesos por dólar. 


\begin{tabular}{|c|c|c|}
\hline & Spot & Futuro \\
\hline Set FX & 3,550 & \\
\hline Cámara de Divisas & 5,845 & \\
\hline AMV & 796 & \\
\hline CRCC & & 9,000 \\
\hline BVC & & 9,000 \\
\hline AMV & & 3,645 \\
\hline Total Costos & 10,191 & 21,645 \\
\hline
\end{tabular}

Tabla 2: Costos variables en pesos colombianos de operación para montos de 250 mil dólares en el mercado Spot (Set-Fx) y el mercado de futuros (X-stream). Algunas cifras han sido aproximadas (ver Apéndice A).
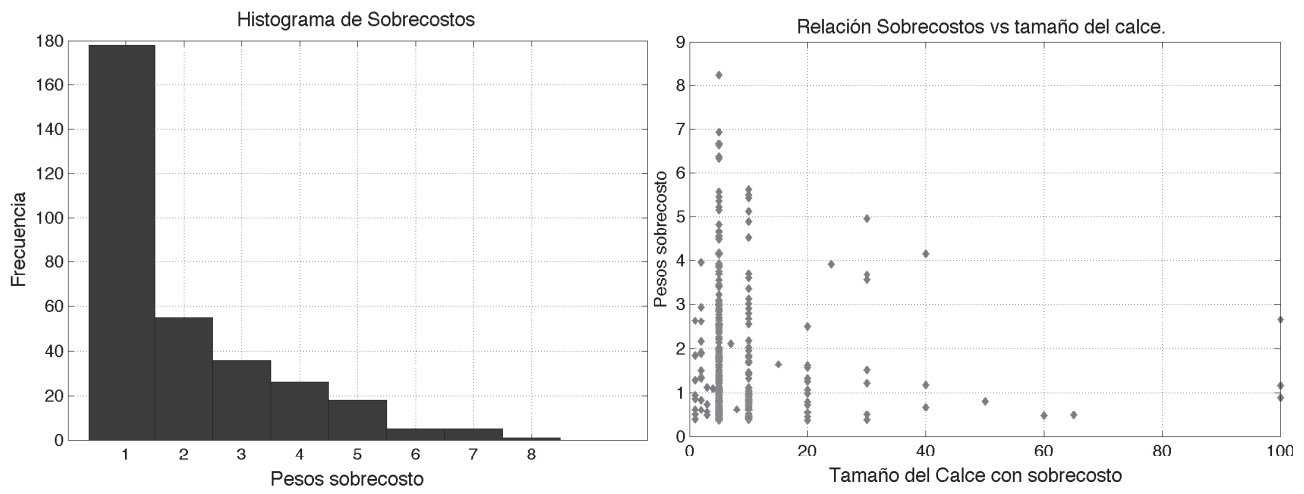

Figura 5: Distribución de sobrecostos para la ventana de tiempo 29/10/2013 - 19/11/2013 sobre los futuros de TRM. Metodología Gamma(7,1.3).

Fuente: cálculos propios.

Para tener una idea del total del sobrecosto, la tabla 5 resume el efecto del total del sobrecosto sobre la ventana estudiada. Para el caso del método Gamma $(7,1.3)$ el total del sobrecosto sobre los 14 días suma 277 millones de pesos.

Utilizando la segunda metodología, MinMax, las estadísticas de los sobrecostos cambian debido a que este método considera escenarios extremos para realizar los calces. Siguiendo nuevamente la tabla 3, el porcentaje de calces que estuvieron por fuera de mercado al considerar las ofertas de compra y venta extremas (ponderadas por volumen del calce) en el intervalo anterior de $15 \mathrm{mi}-$ nutos, fue el 12,18\% de los calces estudiados que totalizaron 9.792 contratos. Bajo esta metodología, el mayor sobrecosto fue de 7,5 pesos por dólar, bastante 


\begin{tabular}{|c|c|c|}
\hline & MinMax (15 min) & Gamma 95\% 5-20 Min \\
\hline Media & 2,07 & 1,91 \\
\hline Desviación & 1,47 & 1,56 \\
\hline Min & 0,40 & 0,36 \\
\hline Max & 7,50 & 8,23 \\
\hline Cant. Fuera mercado (contratos) & 1.193 & 2.871 \\
\hline Cant. Total contratos (contratos) & 9.792 & 9.577 \\
\hline \% contratos por fuera de mercado & $12,18 \%$ & $29,98 \%$ \\
\hline
\end{tabular}

Tabla 3: Estadísticas de los sobrecostos calculadas sobre la ventana de tiempo 29/10/201319/11/2013 acumulando los contratos TRMX13F y TRMZ13F (datos en millones de pesos). Fuente: cálculos propios.

cercano al encontrado con la metodología $\operatorname{Gamma}(7,1.3)$. Siguiendo la tabla 5, el sobrecosto total desciende a 127 millones de pesos, cifra nada despreciable teniendo en cuenta que se está considerando el escenario más extremo posible del libro de órdenes para la realización de la transacción durante los últimos 15 minutos.

El sobrecosto total encontrado puede ser considerado un proxy de la comisión cobrada por los intermediarios financieros a sus clientes si se puede mostrar evidencia que coloque al sobrecosto a favor de las firmas intermediarias y en contra de los clientes. Los participantes de las operaciones del mercado de futuros TRM pueden ser clasificados como: terceros-clientes $(\mathrm{T})$, posición Propia (P) y carteras colectivas (C). La figura 6 muestra la distribución de contrapartes en las operaciones totales estudiadas. Como se observa, Terceros y Posición propia participan casi de manera igualitaria en todas las operaciones. Más aún, Terceros y Posición propia son la combinación que representa el $86 \%$ de los calces del mercado de futuros (tabla 4).

\begin{tabular}{|c|c|}
\hline & Participación(\%) \\
\hline Terceros-Posición Propia & $86 \%$ \\
\hline Carteras-Posición Propia & $7 \%$ \\
\hline Posición Propia-Posición Propia & $7 \%$ \\
\hline
\end{tabular}

Tabla 4: Distribución de los calces en función de las partes involucradas. Ventana de tiempo del 29/10/2013 al 19/11/2013.

Fuente: cálculos propios.

Sin embargo, las operaciones que han sido marcadas como por fuera de mercado por el modelo Gama(5,1.3), cuentan con una distribución de contrapartes muy diferente a la observada en la figura 6. Como se observa en la figura 7, 
calces que tuvieron precios inferiores a los disponibles en el libro de órdenes, tuvieron casi el doble de presencia de intermediarios de mercado beneficiándose del sobrecosto. El mismo comportamiento se evidencia en los calces que fueron marcados por fuera de mercado por estar con precios superiores a los ofrecidos en el libro de órdenes. Los intermediarios tuvieron una presencia casi tres veces mayor que los clientes. Así, se observa que el sobrecosto de las operaciones está a favor de los intermediarios de mercado y en contra de algunos clientes.

Por último, dada la asimetría de información prenegociación que existe en el mercado de futuros de TRM, no es coherente que los terceros estén en posición dominante contra la posición propia. Los calces donde se muestra que los terceros son los beneficiados del sobrecosto, pueden ser mejor entendidos si se acepta la idea de que los intermediarios de mercado no están cumpliendo su obligación de registro de operaciones en menos de 15 minutos después de realizar la transacción. Este punto se investigará en futuros trabajos.

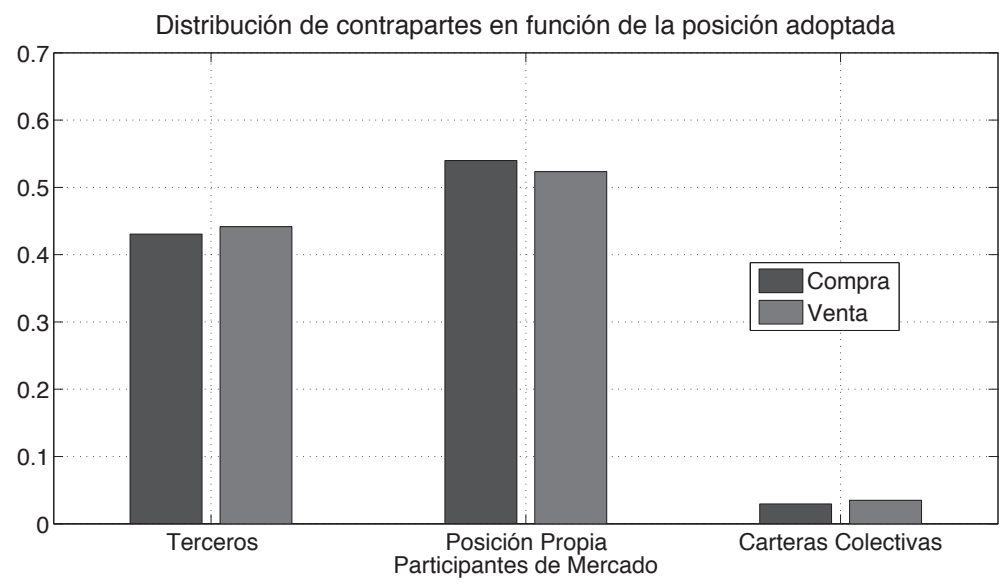

Figura 6: Distribución de contrapartes en función del lado de mercado en el que participaron en las operaciones. Terceros son todos los participantes que no tienen acceso directo al mercado. Posición propia hace referencia a la posición propia de los intermediarios de mercado. Carteras colectivas reúne todas las carteras colectivas participantes. 


\section{Discusión y recomendaciones}

Después de contrastar los resultados observados en las figuras 6 y 7 , se encuentra evidencia de que los registros OTC de operaciones sobre el futuro del TRM están favoreciendo a los agentes informados y desfavoreciendo a los clientes desinformados. Teniendo en cuenta la experiencia internacional, se recomienda que para evitar estos desbalances y distorsiones que podrían frenar el crecimiento de los futuros de TRM en el mediano y largo plazo, se restrinja el tipo de operaciones que pueden realizarse vía la figura de registro.
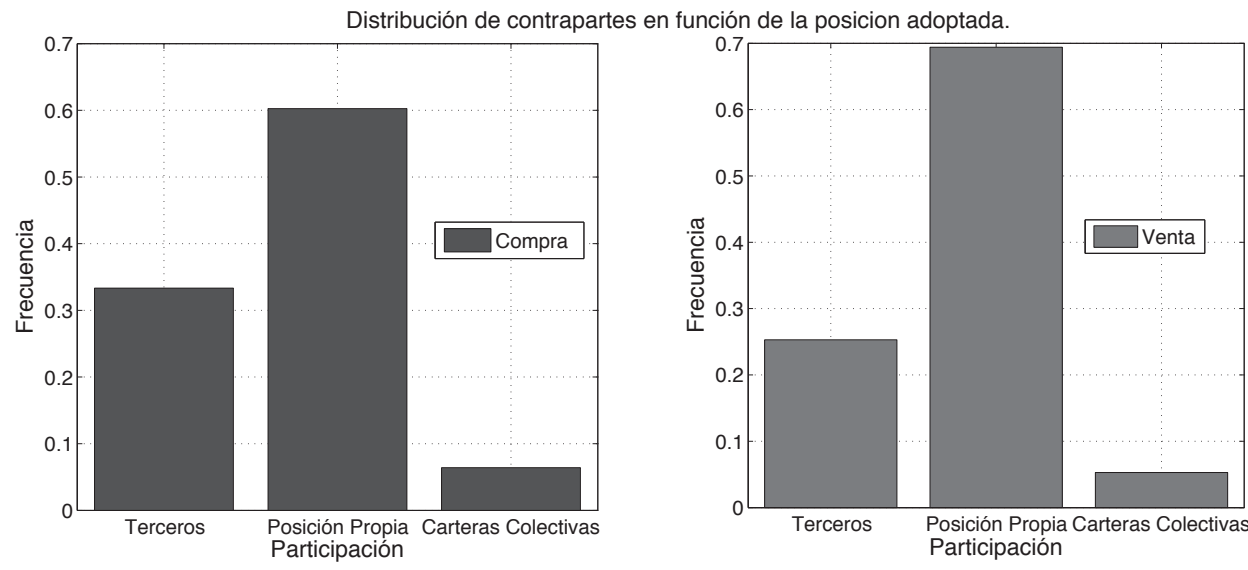

Figura 7: Distribución de contrapartes en función del lado de mercado en el que participaron en las operaciones. (Izquierda) La gráfica muestra la participación en las operaciones que estuvieron por fuera de mercado con precios inferiores a los observados en el libro de órdenes; por tanto, con ventaja para el comprador. (Derecha) Participación en las operaciones que estuvieron por fuera de mercado con precios superiores a los observados en el libro de órdenes; por tanto, con ventaja para el vendedor.

Dado que los clientes están en desventaja en términos de transparencia prenegociación, las transacciones con clientes como contraparte deberían realizarse utilizando el motor de calce de la bolsa como primer canal transaccional. Los registros con clientes solo se utilizarían si no existe oferta de mercado o si el agente intermediario (posición propia) ofrece mejores condiciones que las del mercado para el momento en que se realiza la operación.

Si se incorporara esta recomendación, se garantizaría que los clientes desinformados no estuvieran en desventaja y que la supervisión del mercado de futuros de TRM se simplificara dado que el motor de calce garantiza las mejores condiciones de mercado en las transacciones. 


\begin{tabular}{|c|c|c|}
\hline & MinMax (15 min) & Método Gamma 95\% 5-20 Min \\
\hline TRMX13F & 58 & 160 \\
\hline TRMZ13F & 69 & 117 \\
\hline TOTAL & 127 & 277 \\
\hline
\end{tabular}

Tabla 5: Sobrecostos totales calculados sobre la ventana de tiempo 29/10/2013-19/11/2013 y acumulando los contratos TRMX13F y TRMZ13F (datos en millones de pesos).

Fuente: cálculos propios.

\section{Conclusiones}

La normativa del mercado de derivados estandarizado colombiano permite precisar claramente el nivel de transparencia que debería tener el mercado de futuros de TRM. Para el caso de transparencia prenegociación, el mercado es transparente para los agentes que tienen acceso a la información de los sistemas transaccionales. En términos de transparencia posnegociación, la normatividad obliga a los intermediarios de mercado a registrar sus operaciones realizadas por fuera del motor de calce de la bolsa, en un término no mayor a 15 minutos.

Dado que se asume que los agentes con acceso a la información prenegociación no aceptan precios por fuera de mercado, se planteó la hipótesis de que las transacciones OTC y el posterior registro de los futuros de TRM están siendo usados para generar sobrecostos en los calces con usuarios desinformados.

Para validar la hipótesis planteada, se estudiaron los datos de mercado, libro de órdenes y calces comprendidos entre el 29/10/2013 y el 19/11/2013 utilizando dos metodologías de comparación debido a que la normatividad vigente no define un tiempo exacto que debe transcurrir entre las operaciones y el respectivo registro.

El estudio de la serie de datos arrojó que existe un número no despreciable de operaciones de registro que se están realizando por fuera de mercado en condiciones peores que las que se hubiesen conseguido utilizando el motor de calce de la bolsa.

Dado que el tamaño más probable de operaciones con sobrecosto fue $5 \mathrm{y}$ 10 contratos, se afirma que los sobrecostos se presentan en su mayoría con agentes desinformados que recurren al mercado para negociar posiciones de menor tamaño. 
Esta afirmación se validó encontrando la distribución de contrapartes en las operaciones con sobrecosto después de clasificarlas según la posición adoptada por la posición propia de los intermediarios financieros.

Se concluye, por tanto, que una de las razones para la ausencia de transacciones realizadas por el motor de calce y la proliferación de registros de negociaciones OTC, se debe a la ventaja que explota el intermediario del mercado de futuros frente a las contrapartes desinformadas.

Es importante tener en cuenta que los costos transaccionales de otorgar liquidez por fuera del sistema transaccional regular fueron calculados y tomados en cuenta en el cálculo de las violaciones de los precios de los calces en comparación con los libros de órdenes de los contratos de futuros de TRM.

Este trabajo recomienda que para eliminar la desventaja entre agentes informados y desinformados, se restrinja la realización de registros solamente a operaciones entre agentes informados (posición propia y carteras colectivas). Para el caso de clientes, el regulador debería permitir registros, solamente cuando el libro de órdenes del contrato de futuros de TRM no tuviera la liquidez suficiente, o si la contraparte ofrece mejores condiciones de mercado.

Por último, este trabajo realizó por primera vez para el mercado de derivados estandarizado colombiano, el cáculo del impacto de la asimetría de información cuando se negocia entre agentes informados y desinformados. Este valor fue calculado en un mínimo de 127 millones de pesos y un valor promedio sobre el total del intervalo de 277 millones para los 14 días estudiados. Esta última cifra equivalió a 20 millones de pesos diarios de ineficiencia debido a la posibilidad de registrar operaciones con precios por fuera de mercado. 


\section{Referencias}

Banco de la República (2013). Circular Reglamentaria Externa DODM-317. Bogotá: Banco de la República.

BVC (2013). Circular Única Mercado de Derivados. Recuperado de http://www. bvc.com.co/pps/tibco/portalbvc/Home/Regulacion/Sistemas Administrados/ Derivados?com.tibco.ps.pagesvc.action=updateRenderState\&rp.currentDocu mentID=5d9e2b27_11de9ed172b_-2ca67f000001\&rp.revisionNumber $=1 \&$ rp. attachmentProperty Name $=$ Attachment $\&$ com $. t i b c o . p s . p a g e s v c . t a r g e t$ Page $=1$ f9a1c33_132040fa022_-78750a0a600b\&com.tibco.ps.pagesvc.mode $=$ resource\&rp.redirectPage $=1 \mathrm{f} 9 \mathrm{a} 1 \mathrm{c} 33 \_132040 \mathrm{fa} 022 \_-787 \mathrm{e} 0 \mathrm{a} 0 \mathrm{a} 600 \mathrm{~b}$

Congreso de la República de Colombia (2005). Ley 964 de 2005. Congreso de la República.

EC (2006). Call for Evidence: Pre-and post-trade transparency provisionsof the Markets in Financial Instruments Directive (MiFID) in relation totransactions in classes of financial instruments other than shares. (Inf. Téc.). Brussels: European Commission, Internal Market and Services DG.

Harris, L. (2002). Trading and Exchanges: Market Microstructure for Practitioners. Oxford University Press, USA: Hardcover. Recuperado de http://www.amazon.com/ exec/obidos/redirect?tag=citeulike07-20\&path=ASIN/0195144708

ISDA (2009). Transparency and over-the-counter derivatives: The role of transaction transparency (Inf. Téc.). Recuperado de http://www.isda.org/researchnotes/pdf/ isda-research-notes1.pdf

Superfinanciera (2008). Circular Externa 019 de 2008. Bogotá: Superintendencia Financiera de Colombia. 


\section{A. Apéndice 1}

El cáculo de los costos variables de la tabla 2 fue realizado teniendo en cuenta un monto de transacción de 250 mil dólares y los siguientes supuestos:

\section{DÓLAR SPOT}

\section{- SET-FX}

1. Cargo por transacción realizada en el sistema Dólar Spot y Dólar Next Day (a partir de 501 operaciones - Costo COP 2.300).

2. Cargo por millón de dólares transado por el sistema en el mercado Dólar Spot y Dólar Next Day (entre 0 y 1.500 millones de dólares Costo COP 5.000).

- Cámara de divisas

1. Costo variable mensual de operación por cada punta u operación (desde 0 puntas promedio diario hasta 1.500 puntas promedio diario Costo USD 3,0682). Se consideró como TRM de referencia 1904,98 obtenido como promedio aritmético de la TRM desde el 01/06/2013 hasta 17/11/2013.

- AMV

1. Contribución por número de operaciones (COP 577,77 por operación).

2. Contribución por volumen de operaciones (COP 872,30 por millón de USD transado).

\section{FUTURO TRM}

- Cámara de Riesgos Central de Contraparte

1. Desde 0 hasta 3.000 contratos compensados y liquidados durante un mes calendario. Costo COP 1.800.

- Bolsa de Valores de Colombia

1. Desde 0 hasta 3.000 contratos negociados durante un mes calendario. Costo COP 1.800 . 
- AMV

1. Contribución por número de operaciones (COP 691,57 por operación).

2. Contribución por volumen de operaciones (COP 0,394 por millón transado). Se consideró como TRM de referencia 1904,98 obtenido como promedio aritmético de la TRM desde el 01/06/2013 hasta $17 / 11 / 2013$. 



\title{
Un modelo de creación de mercado con trading de alta frecuencia
}

\author{
Daniel Hernández Hernández \\ Katherine Sánchez Casas ${ }^{* *}$
}

* Docente Centro de Investigación en Matemáticas cimat, Guanajuato (México).dher@ cimat.mx ** Departamento de Estadística Universidad Nacional de Colombia, Bogotá (Colombia). ksanchezc@unal.edu.co

Fecha de recepción: 15 de mayo de 2016.

Fecha de aceptación: 5 de junio de 2016.

Para citar este artículo:

Hernández Hernández, D. y Sánchez Casas, K. (2016). Un modelo de creación de mercado con trading de alta frecuencia. ODEON, 11, pp. 123-142. DOI: https://doi.org/10.18601/17941113.n11.06 
